\title{
Computationally Efficient Robust Chinese Remainder Theorem for Frequency Estimation from Undersampled Waveforms
}

\author{
Xiaoping Li, Wenjie Wang, Bobin Yao, Bin Yang, and Qinye Yin \\ MOE Key Lab for Intelligent Networks and Network Security, Xian Jiaotong University, Xian, Shaanxi 710049, China \\ Correspondence should be addressed to Xiaoping Li; lixiaoping@stu.xjtu.edu.cn
}

Received 28 April 2013; Revised 26 October 2013; Accepted 13 November 2013

Academic Editor: Alexei Mailybaev

Copyright ( 2013 Xiaoping Li et al. This is an open access article distributed under the Creative Commons Attribution License, which permits unrestricted use, distribution, and reproduction in any medium, provided the original work is properly cited.

\begin{abstract}
We present an efficient algorithm based on the robust Chinese remainder theorem (CRT) to perform single frequency determination from multiple undersampled waveforms. The optimal estimate of common remainder in robust CRT, which plays an important role in the final frequency estimation, is first discussed. To avoid the exhausted searching in the optimal estimation, we then provide an improved algorithm with the same performance but less computation. Besides, the sufficient and necessary condition of the robust estimation was proposed. Numerical examples are also provided to verify the effectiveness of the proposed algorithm and related conclusions.
\end{abstract}

\section{Introduction}

Frequency estimation from noisy sinusoid is a hot research topic in engineering, which is applied in almost all engineering fields, such as communications, radar, astronomy, medicine, measurements, and geophysical exploration [1-6]. At present, many of the strategies are based on the Nyquist sampling theorem, for instance, discrete Fourier transform (DFT) algorithm [7-9], the least-error squares algorithm [1013], and equally spaced samples algorithm [14]. However, one of the main drawbacks of these methods is that the sampling frequency is usually proportional to the original frequency, which requires much higher speed analog-todigital convertor. Recently, frequency estimation from undersampled waveforms has been studied in [15-17]. The basic idea of these methods is to fuse multiple remnant information provided by multiple sub-Nyquist sampled sequences. Then the original frequency can be reconstructed by using the Chinese remainder theorem (CRT) [18-20]. The biggest merit of these methods is that they greatly decrease the rigorous requirement for hardware; that is, a very high frequency can be determined by several lower rate sample devices. In addition, the method is also used in synthetic aperture radar $[21,22]$ and wireless sensor network [23].
The problem of frequency determination in undersampled condition is summarized as follows.

Assume that a single frequency signal is modelled as a complex sinusoid form:

$$
s_{0}(t)=A \exp \left(j 2 \pi f_{0} t\right),
$$

where $f_{0}=N \mathrm{~Hz}$ is an unknown frequency we are interested in the signal and it may be high, $A$ is the signal amplitude, and $j=\sqrt{-1}$. In practical applications, noises are unavoidable and thus lead to the received signal having the following expression:

$$
s(t)=A \exp \left(j 2 \pi f_{0} t\right)+w(t),
$$

where $w(t)$ denotes noise.

Let the sampling rates be $M_{1} \mathrm{~Hz}, M_{2} \mathrm{~Hz}, \ldots, M_{L} \mathrm{~Hz}$. Then we can get the corresponding sampled signal as follows:

$$
s_{i}\left(\frac{n}{M_{i}}\right)=A \exp \left(\frac{j 2 \pi N n}{M_{i}}\right)+w\left(\frac{n}{M_{i}}\right), \quad i=1,2, \ldots L .
$$

If the sampling duration is $T$ seconds, then

$$
s_{i}\left(\frac{n}{M_{i}}\right)=A \exp \left(\frac{j 2 \pi N n}{M_{i}}\right)+w\left(\frac{n}{M_{i}}\right), \quad 0 \leq n<T M_{i} .
$$


The $M_{i}$-point DFT of each of the aforementioned sampled signals may provide an estimate of the original frequency. Because $M_{i}$ are much smaller than $N$ in practical applications, the $M_{i}$-point DFT only provides a folded remainder $r_{i}$ of $N$ modulo $M_{i}$. These remainders may be erroneous when the signal is contaminated by the additive noise. So, the above frequency estimation problem is equivalent to determining the original number $N$ from the erroneous remainders $\widehat{r}_{1}$, $\widehat{r}_{2}, \ldots, \widehat{r}_{L}$.

This problem has been studied in [17] where a fast robust CRT algorithm is proposed. However, the number of searches is high and no closed-form solution is provided. It has been validated that this method has a poorer performance than the improved closed-form robust CRT (ICRT), which is proposed in [24]. The latter method improved the performance because of the remainders' proper choosing. However, the estimation of common remainder requires searching, which leads to a high computation complexity. Motivated by this, we propose a computational efficient algorithm which has the same performance as the ICRT method but with much less computation.

The remaining of this paper is organized as follows. In Section 2, we present the CRT and analyse its error sensitivity. In Section 3, we propose the robust CRT frequency estimation algorithm. Besides, we give a sufficient and necessary condition of the robust estimation based on the proposed algorithm. In Section 4, we apply the proposed algorithm for signal in additive noise with low sample rates. We end with concluding remarks in Section 5.

\section{The Error Sensitivity of CRT}

In this section, we first briefly describe the extended CRT where all the moduli have a common factor larger than 1 . We then briefly describe the error sensitivity of CRT.

For convenience, we denote by $\langle x\rangle_{M}$ the remainder of $x$ modulo $M$.

Let $0<M_{1}<M_{2}<\cdots<M_{L}$ be the moduli, and let $r_{1}$, $r_{2}, \ldots, r_{L}$ be the remainders of the positive integer $N$; that is,

$$
N \equiv r_{i} \bmod M_{i}, \quad i=1,2, \ldots, L,
$$

where $0 \leq r_{i}<M_{i}$. It is not hard to see that the original number $N$ can be uniquely reconstructed from its remainders if and only if $N$ is less than the least common multiple of the remainders. In the case when all the moduli are coprime, the CRT has a simple formula. In this paper, we consider the case when any paired moduli $M_{i}$ have the same greatest common remainder (gcd) $M>1$ and the remaining integers factorized by $M$ are coprime integers $\Gamma_{i}$; that is,

$$
r_{i}=\langle N\rangle_{M \Gamma_{i}}, \quad i=1,2, \ldots, L
$$

It follows from (6) that

$$
\left\langle r_{i}\right\rangle_{M}=\langle N\rangle_{M}, \quad i=1,2, \ldots, L .
$$

One can see from (7) that all of the remainder $r_{i}$ moduli $M$ have the same value, which is called the common remainder, denoted by $r^{c}$.
Let $q_{i}=\left(r_{i}-r^{c}\right) / M$, and let $N_{0}=\left(N-r^{c}\right) / M$. Then we obtain from (5) that

$$
N_{0} \equiv q_{i} \bmod \Gamma_{i}
$$

According to the CRT, if and only if $0 \leq N_{0}<\Gamma=\Gamma_{1} \Gamma_{2} \cdots \Gamma_{L}$, $N_{0}$ can be uniquely reconstructed as

$$
N_{0}=\left\langle\sum_{i=1}^{L} \bar{\gamma}_{i} \gamma_{i} q_{i}\right\rangle_{\Gamma},
$$

where $\gamma_{i}=\Gamma / \Gamma_{i}, \bar{\gamma}_{i}$ is the modular multiplicative inverse of $\gamma_{i}$ modulo $\Gamma_{i}$. Therefore, $N$ can be uniquely reconstructed by

$$
N=M N_{0}+r^{c} \text {. }
$$

Now, we consider the case when the remainders have errors.

Let the $i$ th erroneous remainder be

$$
\widehat{r}_{i}=r_{i}+\Delta r_{i}
$$

where $\Delta r_{i}$ denotes the error. In order to reconstruct the original number $N$ from its erroneous remainders $\widehat{r}_{1}, \widehat{r}_{2}, \ldots, \widehat{r}_{L}$, we need to determine $r^{c}$ and $N_{0}$ according to (10). Suppose that the estimate of the common remainder is $\widehat{r}^{c}$. Then $q_{i}$ can be estimated by

$$
\widehat{q}_{i}=\left[\frac{\widehat{r}_{i}-\widehat{r}^{c}}{M}\right]=q_{i}+\left[\frac{\Delta r_{i}-\Delta r^{c}}{M}\right]
$$

where $\Delta r^{c}=\widehat{r}^{c}-r^{c}$ and $[\cdot]$ stands for the rounding integer.

According to (12), if the error of the common remainder $\Delta r^{c}$ is constrained by

$$
\frac{-M}{2}+\Delta r_{i} \leq \Delta r^{c}<\frac{M}{2}+\Delta r_{i}
$$

then $\hat{q}_{i}=q_{i}$. Consequently, $N_{0}$ can be accurately reconstructed. It follows from (10) that the estimate of the original number is

$$
\widehat{N}=M N_{0}+\widehat{r}^{c}
$$

Obviously, the estimate error is $\Delta r^{c}$.

Now, we consider condition (13), which leads to the correct estimate of $N_{0}$. It can be rewritten as

$$
\frac{-M}{2}+\Delta r_{i}+r^{c} \leq \hat{r}^{c}<\frac{M}{2}+\Delta r_{i}+r^{c} .
$$

Note that the fact that $\widehat{r}^{c}$ does not satisfy (15) will lead to the error estimate of $q_{i}$ by (12). As $\bar{\gamma}_{i} \gamma_{i}$ is usually a large integer, this may cause a large error of $N_{0}$. For example, let $N_{0}=51, \Gamma_{1}=11, \Gamma_{2}=13$, and $\Gamma_{3}=17$. Then we have $q_{1}=7$, $q_{2}=12$, and $q_{3}=0$. Suppose that the contaminated data are $\widehat{q}_{1}=6, \widehat{q}_{2}=12$, and $\widehat{q}_{3}=0$. Then $N_{0}$ can be reconstructed as 2661. However, in the case when all errors are the same, the error of reconstruction is reduced significantly. Suppose that the estimate of $q_{i}$ is $\widehat{q}_{i}=q_{i}+\Delta q$ for $i=1,2, \ldots, L$. Then we have $\widehat{N}_{0}=N_{0}+\Delta q$, which means that the estimate error of $N_{0}$ is $\Delta q$. Therefore, the estimate of the common remainder $\widehat{r}^{c}$ is significant to the estimation. 


\section{Robust CRT Frequency Estimation Algorithm}

In this section, we give the optimal estimate of the common remainder firstly; then we propose the efficient frequency estimation algorithm. Before introducing our method, we introduce a kind of circular distance function.

Definition 1. For real numbers $x$ and $y$, the circular distance of $x$ to $y$ for cycle $M$ is defined as

$$
d(x, y \mid M) \stackrel{\Delta}{=} x-y-k_{0} M
$$

where $k_{0}=[(x-y) / M]$ and $M \in \mathbb{R} \backslash\{0\}$.

Putting

$$
\widehat{r}_{i}^{c}=\left\langle\widehat{r}_{i}\right\rangle_{M},
$$

then we can conclude that $\widehat{r}_{i}^{c}$ may be different from each other due to errors $\Delta r_{i}$. The optimal estimate $\widehat{r}^{c}$ is the point which minimizes the summation of the deviation between $\widehat{r}_{i}^{c}$ and variable $x$, where $x \in[0, M)$. Based on the circular distance defined above, we can obtain the summation of deviation as follows:

$$
D(x)=\sum_{i=1}^{L} d^{2}\left(\widehat{r}_{i}^{c}, x \mid M\right)
$$

Then, the optimal estimate $\widehat{r}^{c}$ can be determined by

$$
\widehat{r}^{c}=\arg \min _{0 \leq x<M} D(x) .
$$

Next, we give the optimal estimate in the following theorem.

Theorem 2. The optimal estimate $\widehat{r}^{c}$ in (19) can be determined by

$$
\widehat{r}^{c}=\arg \min _{x \in \Omega} \sum_{i=1}^{L} d^{2}\left(\widehat{r}_{i}^{c}, x \mid M\right),
$$

where $\Omega=\left\{\left\langle(1 / L) \sum_{i=1}^{L} \widehat{r}_{i}-(M / L) k\right\rangle_{M}, k=0,1, \ldots, L-1\right\}$.

Proof. According to the definition of the circular distance, we have

$$
d\left(\widehat{r}_{i}^{c}, x \mid M\right)=d\left(\widehat{r}_{i}, x \mid M\right)
$$

Let $f_{i}(x)=d^{2}\left(\widehat{r}_{i}, x \mid M\right)$. One can easily see that $f_{i}(x)$ is continuous in field of real number. Now, we consider the differentiability of $f_{i}(x)$ at $x_{t}$ which satisfies

$$
d\left(\widehat{r}_{i}, x_{t} \mid M\right)=\frac{-M}{2} .
$$

For $x \in\left(x_{t}-\epsilon, x_{t}\right)$, where $\epsilon$ is a small positive number, we have $d\left(\widehat{r}_{i}, x \mid M\right)=x_{t}-M / 2-x$. It follows that the left-sided derivative of $f_{i}(x)$ at $x_{t}$ is $M$.

For $x \in\left(x_{t}, x_{t}+\epsilon\right)$, we obtain $d\left(\widehat{r}_{i}, x M\right)=x_{t}+M / 2-x$. It follows that the right-sided derivative of $f_{i}(x)$ at $x_{t}$ is $-M$. Thus, $f_{i}(x)$ is not differentiable at $x_{t}$.
Let $f(x)=\sum_{i=1}^{L} d^{2}\left(\widehat{r}_{i}, x \mid M\right)$. Then we can obtain

$$
f_{+}^{\prime}\left(x_{t}\right)-f_{-}^{\prime}\left(x_{t}\right)=-2 k M<0,
$$

where $k$ is the number of remainders satisfying (22). Thus, $f(x)$ does not have a local minimum value at $x_{t}$. Note that $f(x)$ is differentiable at all $x \in \mathbb{R} \backslash\left\{x_{t}\right\}$, so the minimum point must satisfy $f^{\prime}(x)=0$, that is, $\widehat{r}^{c}$ in (19), such that

$$
\left(\sum_{i=1}^{L} d^{2}\left(\widehat{r}_{i}, x \mid M\right)\right)^{\prime}=0
$$

After equating the result, we obtain

$$
x=\frac{1}{L} \sum_{i=1}^{L} \widehat{r}_{i}-\frac{M}{L} \sum_{i=1}^{L} k_{i}
$$

Since $\sum_{i=1}^{L} k_{i} \in \mathbb{Z}$ and $\widehat{r}^{c} \in[0, M)$, it follows that $\widehat{r}^{c}$ belongs to

$$
\Omega=\left\{\left\langle\frac{1}{L} \sum_{i=1}^{L} \widehat{r}_{i}-\frac{M}{L} k\right\rangle_{M}, k=0,1, \ldots, L-1\right\} .
$$

Hence, the optimal estimate $\widehat{r}^{c}$ in (19) can be simplified as

$$
\widehat{r}^{c}=\arg \min _{x \in \Omega} D(x) .
$$

Combining (26) and (27), we can draw the conclusion.

Theorem 2 tells us that the estimate of common remainder belongs to the set $\Omega$. It is not difficult to find that the cardinality of the set equals the number of the remainders. So, the number of searches is reduced sharply when compared with other searching methods. After optimal estimate $\widehat{r}^{c}$ is determined, the original frequency can be reconstructed. For convenience, we conclude the proposed efficient algorithm in Algorithm 1.

Based on the proposed algorithm, we have a sufficient and necessary condition of the robust estimate as shown in Algorithm 1

Theorem 3. Let $\overline{\Delta r}=(1 / L) \sum_{i=1}^{L} \Delta r_{i}$. Assume that $|\overline{\Delta r}|<M / 2$ and $\left|\Delta r_{i}-\overline{\Delta r}\right|<M / 2 . N$ is a real number in the range $[M, M(\Gamma-1))$. Then

$$
\widehat{N}-N=\overline{\Delta r}
$$

holds if and only if

$$
L_{0}=\left\langle\sum_{i=1}^{L} q_{i}\right\rangle_{M}
$$

Proof. We proof the sufficiency.

From $(* *)$, we have

$$
\widehat{r}^{c}=\left\langle\frac{1}{L} \sum_{i=1}^{L} \widehat{r}_{i}-\frac{M}{L} £_{0}\right\rangle_{M}=\left\langle r^{c}+\overline{\Delta r}\right\rangle_{M} .
$$


Step 1. From contaminated remainders $\widehat{r}_{i}$, calculate $L_{0}$ :

$$
L_{0}=\arg \min _{k=0,1, \ldots, L-1} \sum_{i=1}^{L} d^{2}\left(\widehat{r}_{i}, \frac{1}{L} \sum_{i=1}^{L} \widehat{r}_{i}-\frac{M}{L} k \mid M\right)
$$

Step 2. Calculate $\widehat{r}^{c}$ :

$$
\widehat{r}^{c}=\left\langle\frac{1}{L} \sum_{i=1}^{L} \widehat{r}_{i}-\frac{M}{L} L_{0}\right\rangle_{M} .
$$

Step 3. Calculate $\widehat{q}_{i}(i=1,2, \ldots, L)$ by (12).

Step 4. Calculate $\widehat{N}_{0}$ :

$$
\widehat{N}_{0}=\left\langle\sum_{i=1}^{L} \bar{\gamma}_{i} \gamma_{i} \widehat{q}_{i}\right\rangle_{M}
$$

Step 5. Calculate $\widehat{N}$ :

$$
\widehat{N}=M \widehat{N}_{0}+\widehat{r}^{c}
$$

Algorithm 1: Summary of the proposed algorithm.

Since $|\overline{\Delta r}|<M / 2$ and $0 \leq r^{c}<M$, it follows that

$$
\frac{-M}{2} \leq r^{c}+\overline{\Delta r}<\frac{3 M}{2} .
$$

We make this into three cases as follows.

Case A. $\left(-M / 2 \leq r^{c}+\overline{\Delta r}<0\right)$.

According to (30), we obtain $\widehat{r}^{c}=r^{c}+\overline{\Delta r}+M$. It follows from (12) that $\widehat{q}_{i}=q_{i}-1$ for $i=1,2, \ldots, L$. From (9), we have

$$
\widehat{N}_{0}=\left\langle\sum_{i=1}^{L} \bar{\gamma}_{i} \gamma_{i}\left(q_{i}-1\right)\right\rangle_{M}=N_{0}-1 .
$$

Here, we use the conclusion that $\left\langle\sum_{i=1}^{L} \bar{\gamma}_{i} \gamma_{i}\right\rangle_{M}=1$. Consequently,

$$
\widehat{N}=M\left(N_{0}-1\right)+\widehat{r}^{c}=N+\overline{\Delta r}
$$

Case B. $\left(0 \leq r^{c}+\overline{\Delta r}<M\right)$.

According to (30), we obtain $\widehat{r}^{c}=r^{c}+\overline{\Delta r}$. It follows from (12) that $\widehat{q}_{i}=q_{i}$. By using CRT, we have $\widehat{N}_{0}=N_{0}$. Consequently, $\widehat{N}=M N_{0}+\widehat{r}^{c}=N+\overline{\Delta r}$.

Case C. $\left(M \leq r^{c}+\overline{\Delta r}<3 M / 2\right)$.

According to (30), we obtain $\widehat{r}^{c}=r^{c}+\overline{\Delta r}-M$. It follows from (12) that $\widehat{q}_{i}=q_{i}+1$. From (9), we have $\widehat{N}_{0}=N_{0}+1$. Consequently, $\widehat{N}=N+\overline{\Delta r}$. This completes the proof of the sufficiency.

We then proof the necessity.

Combination of $(10)$ and $(* * * *)$ yields

$$
\widehat{N}-N=M\left(\widehat{N}_{0}-N_{0}\right)+\left(\widehat{r}^{c}-r^{c}\right)=\overline{\Delta r} .
$$

This leads to

$$
\left|M\left(\widehat{N}_{0}-N_{0}\right)+\left(\widehat{r}^{c}-r^{c}\right)\right|<\frac{M}{2} .
$$

Since $-M<\widehat{r}^{c}-r^{c}<M$, it follows that

$$
\left|\widehat{N}_{0}-N_{0}\right|<\frac{3}{2}
$$

Note that both of $\widehat{N}_{0}$ and $N_{0}$ are integers; we have three possible cases: $\widehat{N}_{0}-N_{0}=-1, \widehat{N}_{0}=N_{0}$, and $\widehat{N}_{0}-N_{0}=1$.

Case A. $\left(\widehat{N}_{0}-N_{0}=-1\right)$.

By (34), we have $\widehat{r}^{c}-r^{c}-M=\overline{\Delta r}$. That is, $\widehat{r}^{c}=r^{c}+\overline{\Delta r}+M$.

Case B. $\left(\widehat{N}_{0}=N_{0}\right)$.

By (34), we have $\widehat{r}^{c}=r^{c}+\overline{\Delta r}$.

Case C. $\left(\widehat{N}_{0}-N_{0}=1\right)$.

By (34), we have $\widehat{r}^{c}-r^{c}+M=\overline{\Delta r}$. That is, $\widehat{r}^{c}=r^{c}+\overline{\Delta r}-M$. Therefore, we have

$$
\widehat{r}^{c}=\left\langle r^{c}+\overline{\Delta r}\right\rangle_{M} .
$$

Combing $(* *)$ and (37), we can draw the conclusion.

\section{Simulation Results}

In the simulations, the coprime numbers from $\Gamma_{1}$ to $\Gamma_{7}$ are $5,7,11,13,19,23$, and 29, respectively. Two possibilities of $M$ are considered: $M=100$ and $M=200$. The frequencies which are to be estimated is taken integers randomly and uniformly distributed in the range $\left(M \Gamma / \Gamma_{1}, M \Gamma\right)$ for each $M$. The additive noise $w(t)$ in (4) is additive white Gauss noise. The sampling frequencies are $M \Gamma_{i}$, which are much smaller than the unknown frequencies. The observation of the time duration is $1 \mathrm{~s}$, and the number of the trials is 10000 for each signal-to-noise ratio (SNR).

We compare the proposed method with the ICRT algorithm which is the most effective one of the existing methods. To assess the performance, we take the trail-fail rate (TFR) 


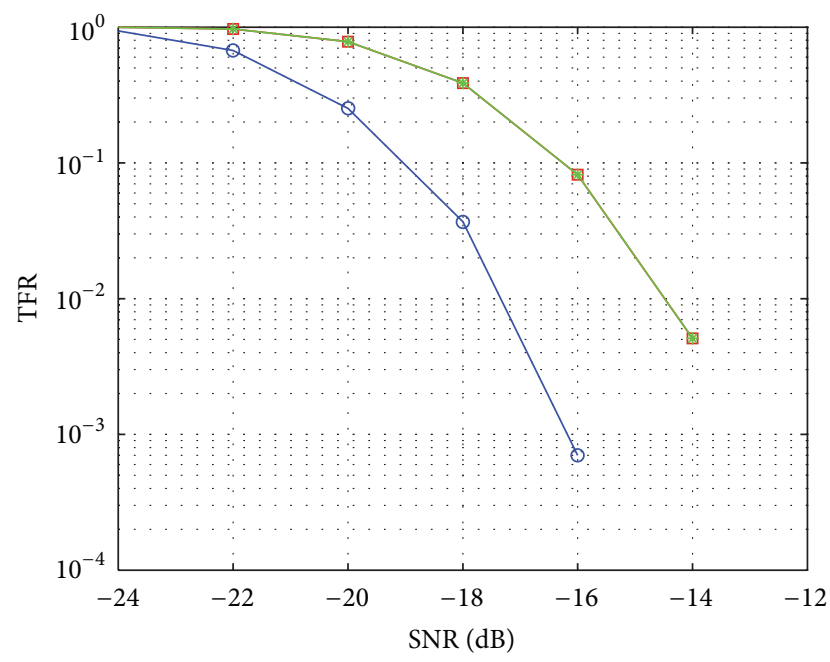

$\square$ Proposed algorithm $(M=100) \rightarrow$ Proposed algorithm $(M=200)$ * ICRT algorithm $(M=100) \quad$ - ICRT algorithm $(M=200)$

FIGURE 1: TFR versus SNR.

as the measurement. In each trial, if the estimate of the frequency is

$$
\left|\widehat{f}_{0}-f_{0}\right|<\frac{M}{2}
$$

then the trial passed; otherwise, the trail failed.

Figure 1 gives the curve of the TFR on different SNR. It shows that the probabilities of robust estimation are almost equal when the two methods have the same $M$. That is to say, the two methods have nearly the same performance. The figure also shows that the performance of the estimation is better when $M=200$ than that when $M=100$, which is in accordance with the theory. From (13) we can conclude that the larger $M$ can lead to the larger error tolerance.

Figure 2 presents the curve of the total running time over the number of remainders. The two methods are carried out under the same operational conditions. One can see that our proposed algorithm has nearly equal time no matter $L$ is, while the ICRT algorithm has essentially linear running time. This is because the number of searches for the common remainder is $L$ for the proposed algorithm. However, the number of searches is $M / \epsilon$ for the ICRT method, where $\epsilon$ is the searching step. In this simulation, the searching step is $\epsilon=1$, and $M=100$.

Figure 3 gives the robust estimation probability of the common remainder for searching methods when $\epsilon$ is 10 , 1 , and 0.1 , respectively. It shows that the estimate of the common remainder can achieve an approximation of the optimal estimate when $\epsilon$ is small enough. Clearly, decreasing the searching step can improve the performance at the cost of increasing the computation amount.

\section{Conclusions}

In this paper, we have proposed an efficient algorithm based on the robust CRT to perform single frequency estimation

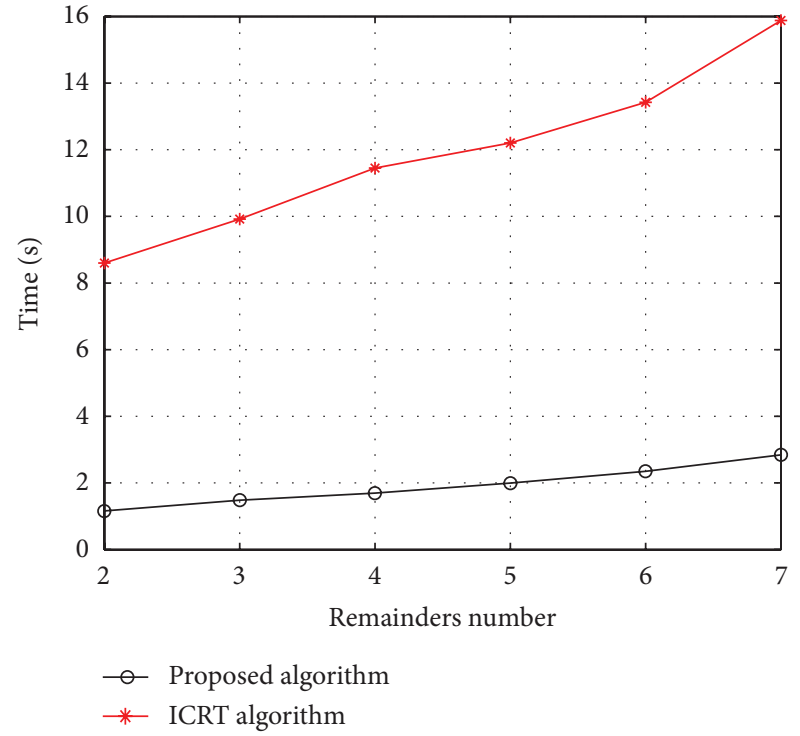

FIGURE 2: Total running time versus remainders number.

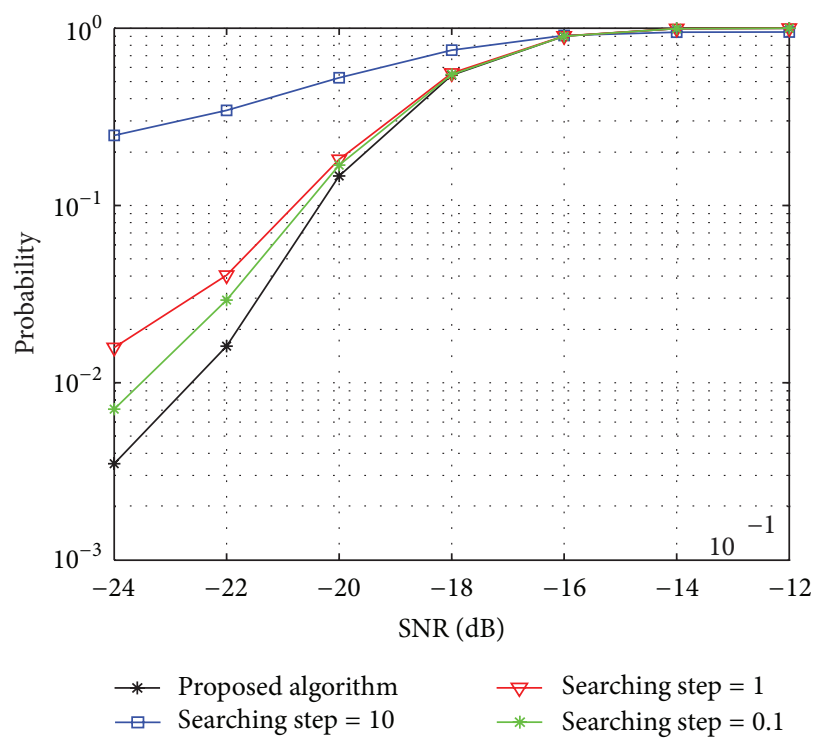

FIGURE 3: Robust estimation probability of common remainder versus SNR.

from multiple undersampled waveforms. We transformed the problem into the robust CRT firstly. Then we presented the optimal estimate of the common remainder and the original frequency. Based on the proposed algorithm, we presented the sufficient and necessary condition of the robust estimation. We finally applied the efficient algorithm to estimate the original frequency from multiple undersampled waveforms. Compared with the improved robust CRT algorithm, it has nearly the same performance but less computation. After using the undersampled waveforms, the sampling rate reduced sharply. 


\section{Acknowledgments}

The authors would like to thank the referee for his (her) valuable comments and suggestions which improved this work to a great extent. This work was supported by the National Natural Science Foundation of China (Grants nos. 61172092, 61302069) and the Research Fund for the Doctoral Programs of Higher Education of China (no. 20130201110014).

\section{References}

[1] B. G. Quinn and E. J. Hannan, The Estimation and Tracking of Frequency, Cambridge University Press, New York, NY, USA, 2001.

[2] C. Gottschlich, "Curved-region-based ridge frequency estimation and curved gabor filters for fingerprint image enhancement," IEEE Transactions on Image Processing, vol. 21, no. 4, pp. 2220-2227, 2012.

[3] E. Lavopa, P. Zanchetta, M. Sumner, and F. Cupertino, "Realtime estimation of fundamental frequency and harmonics for active shunt power filters in aircraft electrical systems," IEEE Transactions on Industrial Electronics, vol. 56, no. 8, pp. 28752884, 2009.

[4] B. Yegnanarayana and K. S. R. Murty, "Event-based instantaneous fundamental frequency estimation from speech signals," IEEE Transactions on Audio, Speech and Language Processing, vol. 17, no. 4, pp. 614-624, 2009.

[5] I. Orović, S. Stanković, T. Thayaparan, and L. J. Stanković, "Multiwindow S-method for instantaneous frequency estimation and its application in radar signal analysis," IET Signal Processing, vol. 4, no. 4, pp. 363-370, 2010.

[6] L. Yang, G. Ren, and Z. Qiu, "A novel doppler frequency offset estimation method for DVB-T system in HST environment," IEEE Transactions on Broadcasting, vol. 58, no. 1, pp. 139-143, 2012.

[7] T. Grandke, "Interpolation algorithms for discrete fourier transforms of weighted signals," IEEE Transactions on Instrumentation and Measurement, vol. 32, no. 2, pp. 350-355, 1983.

[8] Ç. Candan, "A method for fine resolution frequency estimation from three DFT samples," IEEE Signal Processing Letters, vol. 18, no. 6, pp. 351-354, 2011.

[9] I. Djurović and V. V. Lukin, "Estimation of single-tone signal frequency by using the L-DFT,' Signal Processing, vol. 87, no. 6, pp. 1537-1544, 2007.

[10] M. M. Giray and M. S. Sachdev, "Off-nominal frequency measurements in electric power systems," IEEE Transactions on Power Delivery, vol. 4, no. 3, pp. 1573-1578, 1989.

[11] M. D. Kušljević, J. J. Tomić, and L. D. Jovanović, "Frequency estimation of three-phase power system using weighted-leastsquare algorithm and adaptive FIR filtering," IEEE Transactions on Instrumentation and Measurement, vol. 59, no. 2, pp. 322-329, 2010.

[12] A. K. Pradhan, A. Routray, and A. Basak, "Power system frequency estimation using least mean square technique," IEEE Transactions on Power Delivery, vol. 20, no. 3, pp. 1812-1816, 2005.

[13] F. K. W. Chan, H. C. So, W. H. Lau, and C. F. Chan, "Structured total least squares approach for efficient frequency estimation," Signal Processing, vol. 91, no. 4, pp. 1043-1047, 2011.

[14] D. N. Vizireanu, "A fast, simple and accurate time-varying frequency estimation method for single-phase electric power systems," Measurement, vol. 45, no. 5, pp. 1331-1333, 2012.
[15] M. D. Zoltowski and C. P. Mathews, "Real-time frequency and 2-D angle estimation with sub-Nyquistspatio-temporal sampling," IEEE Transactions on Signal Processing, vol. 42, no. 10, pp. 2781-2794, 1994.

[16] D. L. Donoho and J. Tanner, "Precise undersampling theorems," Proceedings of the IEEE, vol. 98, no. 6, pp. 913-924, 2010.

[17] X. Li, H. Liang, and X.-G. Xia, "A robust Chinese remainder theorem with its applications in frequency estimation from undersampled waveforms," IEEE Transactions on Signal Processing, vol. 57, no. 11, pp. 4314-4322, 2009.

[18] B. Krishna, H. C. Krishna, and K. Y. Lin, Computational Number Theory and Digital Signal Processing: Fast Algorithms and Error Control Techniques, CRC, Boca Raton, Fla, USA, 1994.

[19] C. Ding, D. Pei, and A. Salomaa, Chinese Remainder Theorem: Applications in Computing, Coding, Cryptography, World Scientific, Singapore, 1999.

[20] K. H. Rosen, Elementary Number Theory and Its Applications, Addison-Wesley, Mass, 5th edition, 2010.

[21] G. Li, J. Xu, Y.-N. Peng, and N.-G. Xia, "Bistatic linear antenna array SAR for moving target detection, location, and imaging with two passive airborne radars," IEEE Transactions on Geoscience and Remote Sensing, vol. 45, no. 3, pp. 554-565, 2007.

[22] Z. H. Yuan, Y. k. Deng, F. Li et al., "Multichannel InSAR DEM reconstruction through improved closed-form robust Chinese remainder theorem," IEEE Geoscience and Remote Sensing Letters, 2013.

[23] C. Wang, Q. Yin, and H. Chen, "Robust chinese remainder theorem ranging method based on dual-frequency measurements," IEEE Transactions on Vehicular Technology, vol. 60, no. 8, pp. 4094-4099, 2011.

[24] W. Wang and X.-G. Xia, "A closed-form robust Chinese remainder theorem and its performance analysis," IEEE Transactions on Signal Processing, vol. 58, no. 11, pp. 5655-5666, 2010. 


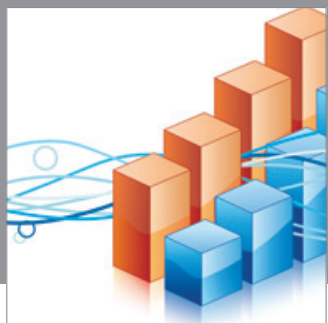

Advances in

Operations Research

mansans

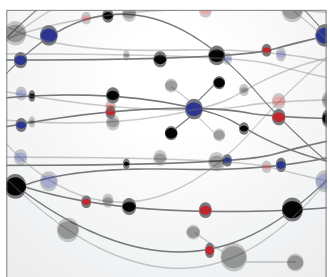

The Scientific World Journal
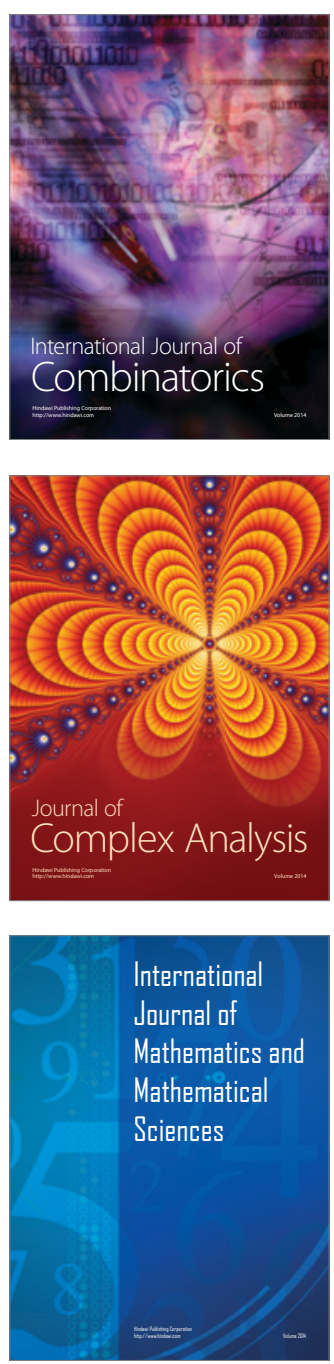
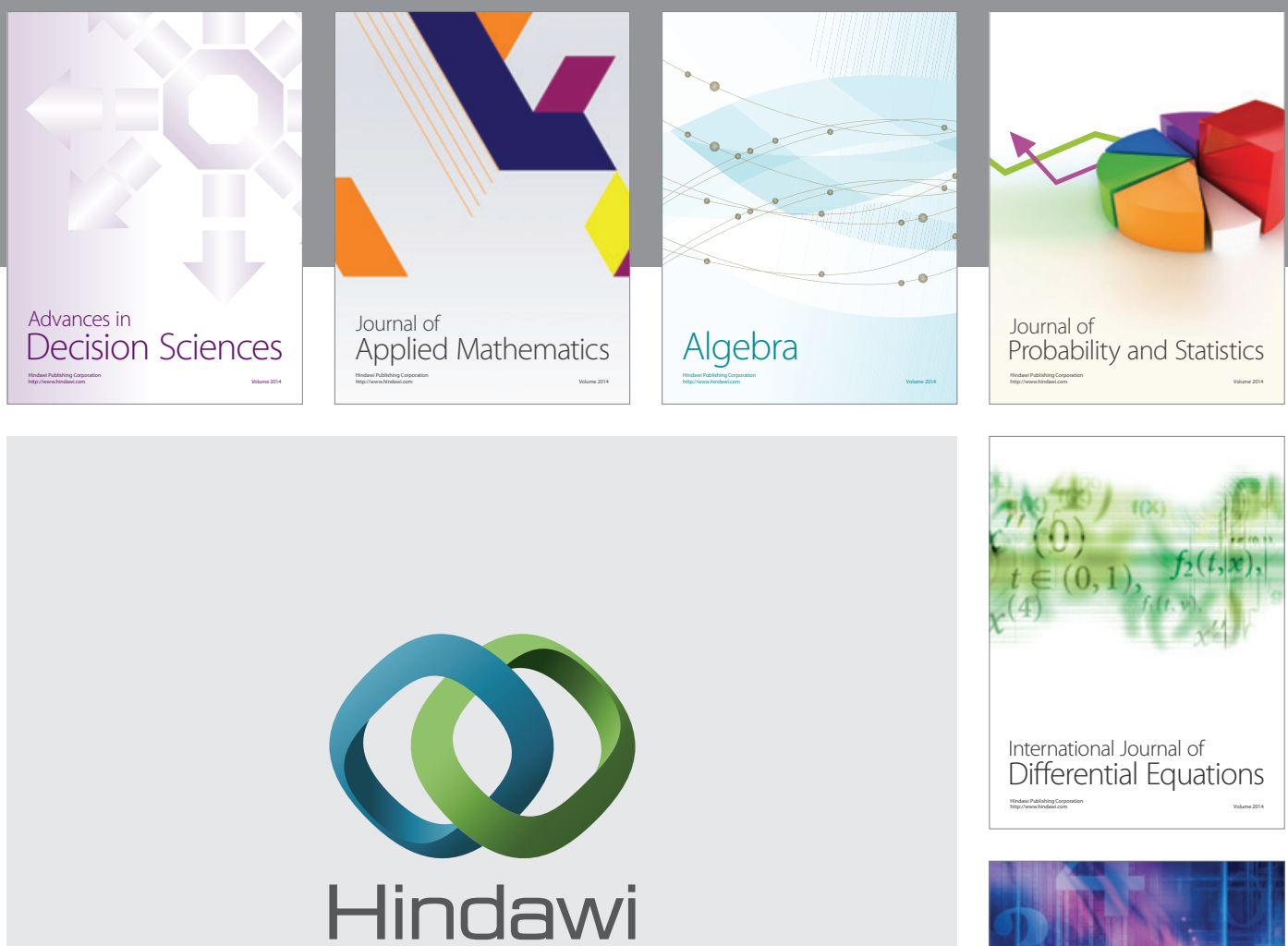

Submit your manuscripts at http://www.hindawi.com
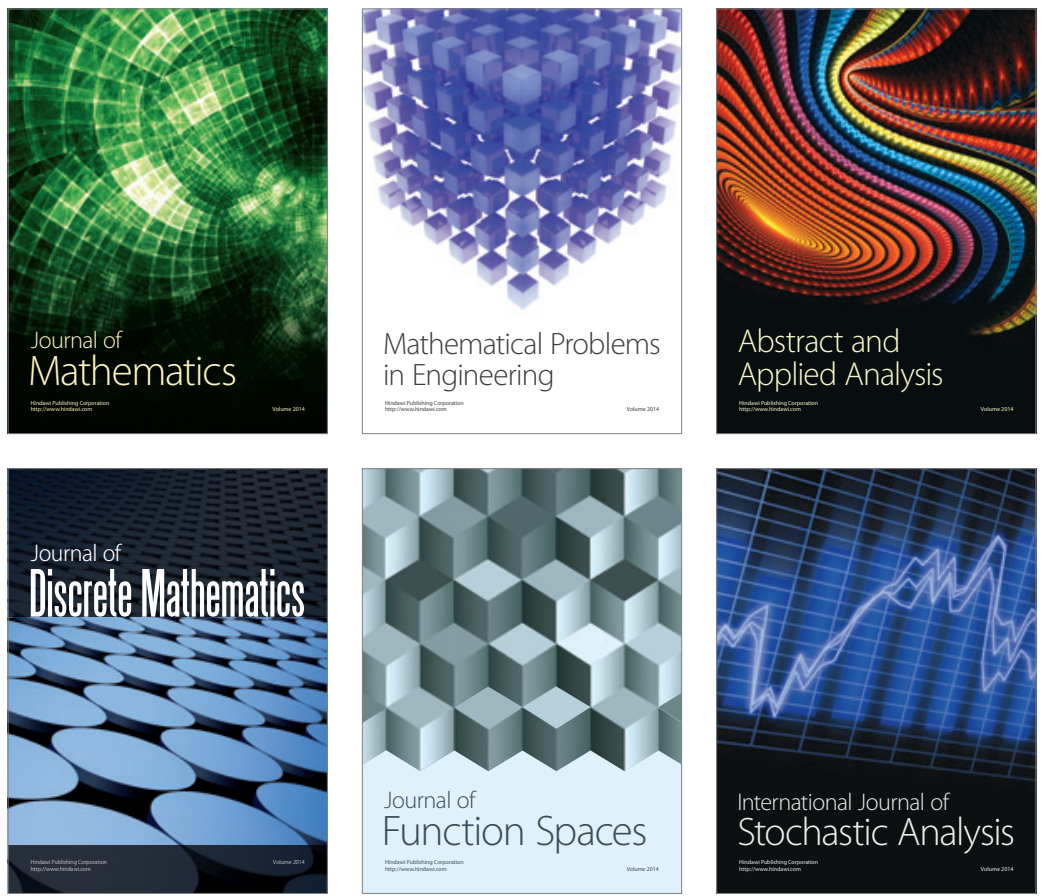

Journal of

Function Spaces

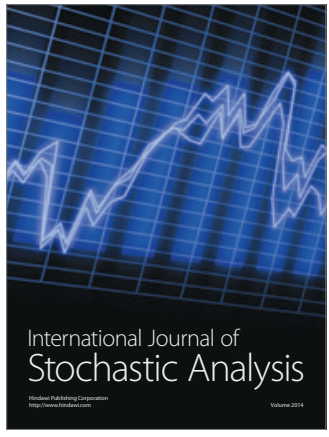

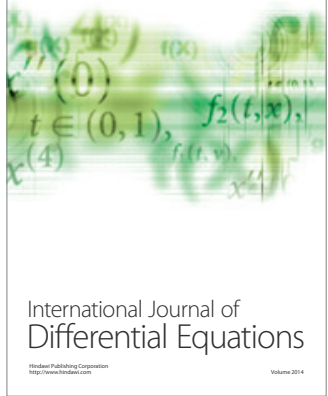
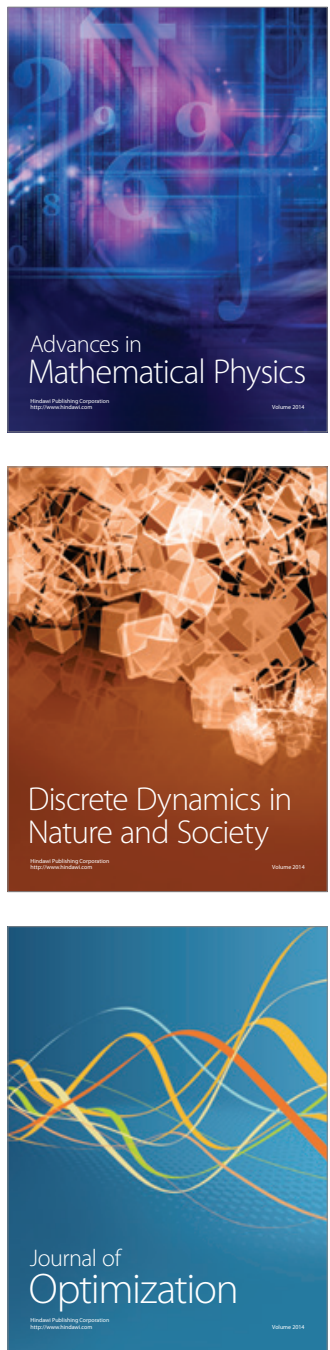